The following paper posted here is not the official IEEE published version. The final published version of this paper can be found in Proceedings: International Parallel and Distributed Processing Symposium: April 15-19, 2002, Ft. Lauderdale, Florida, USA: pp.186-196

Copyright @ 2000 IEEE.

Personal use of this material is permitted. However, permission to reprint/republish this material for advertising or promotional purposes or for creating new collective works for resale or redistribution to servers or lists, or to reuse any copyrighted component of this work in other works must be obtained from the IEEE. 


\title{
Monte Carlo Simulation of HIV-1 Evolution in Response to Selection by Antibodies
}

\author{
Jack da Silva \\ North Carolina Supercomputing Center, MCNC, Research Triangle Park, NC; \\ jdasilva@ncsc.org \\ Austin Hughes \\ Department of Biology, University of South Carolina, Columbia, SC; austin@biol.sc.edu
}

\begin{abstract}
The persistence of human immunodeficiency virus type 1 (HIV-1) infection may be in part attributed to its ability to evolve to evade neutralizing antibody (NAb) surveillance. We have tested the prediction that positive selection of V3 is positively correlated with the strength of a patient's NAb response by analyzing a data set containing both sequences from the principal neutralizing domain of HIV-1 (V3) and measures of the strength of the NAb responses of several patients. Only viral sequences from the patient with the strongest NAb response exhibited evidence of significant positive selection. To investigate the nature of selection by NAbs, we simulated the evolution of V3 at the nucleotide level. Two forms of plausible NAb selection were used: negative frequency-dependent selection and negative viral-age-dependent selection. Assuming negative age-dependent selection rather than negative frequency-dependent selection better simulated the temporal pattern of $\mathrm{V} 3$ variation from the patient with the strongest $\mathrm{NAb}$ response.
\end{abstract}

\section{Introduction}

The persistence of human immunodeficiency virus type 1 (HIV-1) infection may be attributed to the virus's ability to evade immune surveillance (Desrosiers 1999). Viral infection persists, even in the face of an initially vigorous cytotoxic T cell (CTL) response, apparently because HIV-1 is able to adapt to CTL surveillance through the evolution of CTL-escape variants (e.g., McMichael and Phillips 1997; da Silva and Hughes 1999; Evans, O'Connor et al. 1999). It is not clear, however, why even a strong neutralizing antibody (NAb) response is unable to clear the virus. One of the main targets of NAbs is the viral exterior envelope glycoprotein, gp120, which is situated on the virion surface and binds to host-cell chemokine coreceptor molecules during cell entry (Profy, Salinas et al. 1990). Although high levels of antibodies are made against gp120, most are not neutralizing or only weakly neutralizing (Desrosiers 1999). The inability of antibodies to easily neutralize virions may be explained in part by the structure of the core of the oligomeric form of gp120 on intact virions (Fouts, Binley et al. 1997; Kwong, Wyatt et al. 1998).

The 32-amino acid third variable loop (V3) of gp120 has been the focus of much research because of its importance as a NAb target (e.g., Spenlehauer, Saragosti et al. 1998; Nyambi, Mbah et al. 2000), as a determinant of host-cell tropism (e.g., Speck, Wehrly et al. 1997), and because variation within this region may be related to the rate of disease progression (Viscidi 1999; Wolinsky and Learn 1999). Yet, neither the cause of variation nor the relationship between variation and disease progression has been well established. Because V3 is involved in determining chemokine coreceptor usage by the virus, which determines cell tropism (e.g., Speck, Wehrly et al. 1997), it has been hypothesized that adaptation by the virus to a variety of cell types causes diversification of V3 (Weiss 1996; Speck, Wehrly et al. 1997). A competing hypothesis states that continuous selection of escape mutants by NAbs increases variation in V3 (Bonhoeffer, Holmes et al. 1995; Halapi, Leitner et al. 1997; Liu, Schacker et al. 1997). These hypotheses have been difficult to test because selection of $\mathrm{V} 3$ for neutralization resistance may change coreceptor usage (McKnight, Weiss et al. 1995). 
V3 is a known target of monoclonal NAbs (Spenlehauer, Saragosti et al. 1998; Nyambi, Mbah et al. 2000), and several NAb selection studies in vitro (McKnight, Weiss et al. 1995; Schonning, Jansson et al. 1996; Yoshida, Nakamura et al. 1997; Inouye, Cherry et al. 1998) and in vivo using animal models (Cheng-Mayer, Brown et al. 1999; Etemad-Moghadam, Sun et al. 1999) have reported amino acid replacements in V3 that affect neutralization. However, it remains to be demonstrated that HIV-1 V3 variation in humans is the result of in vivo selection by NAbs.

Systems subject to apparently random perturbations, such as the error-prone reverse transcription of RNA and replication of DNA, can be modeled using Monte Carlo techniques, in which a pseudorandom number generator is used to simulate random events (Haefner 1996). This approach has been used to examine potential immune selection of HIV-1 at the molecular genetic level (Wiener 1996), showing that such selection can be detected by analyzing sequence data for evidence of positive selection. However, only one form of positive selection, frequencydependent selection, was modeled. In this type of selection, the fitness of an antigenic variant is inversely related to its relative frequency, as would be expected if immune surveillance were directed predominantly at the most common variants. However, antibody selection likely involves a time lag between the emergence of resistant variants and a neutralizing response to these variants, as has been shown for chimpanzees naturally infected with simian immunodeficiency virus (SIVcpz) (Nyambi, Lewi et al. 1997) and humans infected with HIV-1 (Albert, Abrahamsson et al. 1990; Bradney, Scheer et al. 1999). Furthermore, because of computer resource limitations, unrealistic viral population sizes and mutation rates were used in simulations. Our aim was to use Monte Carlo techniques and realistic parameter values to simulate antibody selection of V3 and compare the resulting temporal patterns of V3 variation to the patterns determined empirically. This will help determine whether antibody selection is a plausible explanation of V3 variation, and, if so, what form of selection is likely operating. The form of selection will reflect the mode and tempo of antibody surveillance, knowledge of which should help to predict the evolutionary responses of HIV-1 to antibody vaccines.

\section{Methods}

\section{Nucleotide Substitution Distances}

Variation in V3 was measured by estimating mean synonymous and nonsynonymous nucleotide substitution distances between pairs of sequences. The mean number of synonymous substitutions per potentially synonymous site $\left(d_{\mathrm{S}}\right)$ and the mean number of nonsynonymous substitutions per potentially nonsynonymous site $\left(d_{\mathrm{N}}\right)$ between all possible pairs of sequences and their variances are estimated using well-established algorithms (Nei and Gojobori 1986; Nei and Jin 1989). $d_{\mathrm{S}}$ and $d_{\mathrm{N}}$ were corrected for multiple (and therefore unobserved) nucleotide changes at a single site by assuming that any nucleotide changes to any other nucleotide with equal probability across all sites (Jukes and Cantor 1969). In the absence of selection for amino acid replacement $d_{\mathrm{N}}$ is expected to equal $d_{\mathrm{S}}$. However, with positive selection, $d_{\mathrm{N}}$ is expected to exceed $d_{\mathrm{S}}$. The statistical null hypothesis $d_{\mathrm{S}}=d_{\mathrm{N}}$ is tested by computing the $Z$-score:

$$
\frac{d \mathrm{~N} d \mathrm{~s}}{\sqrt{V(d \mathrm{~N}) \quad V(d \mathrm{~s})}},
$$

where $\mathrm{V}\left(d_{\mathrm{S}}\right)$ and $\mathrm{V}\left(d_{\mathrm{N}}\right)$ are the variances of $d_{\mathrm{S}}$ and $d_{\mathrm{N}}$, respectively. This value is compared to critical values from the $t$-distribution at 5, 1 and $0.1 \%$ significance and with infinite degrees of freedom (Kumar, Tamura et al. 1993). 


\section{Analysis of Empirical Data}

In a preliminary test of the hypothesis that HIV-1 V3 is under selection by NAbs, we tested the prediction that V3 variation is correlated with the strength of a patient's NAb response and that this variation results from positive selection. The dataset, consisting of sequences from the V3 region sampled from six patients, is the result of a longitudinal study of the relationship between CTL selection and disease progression (Wolinsky, Korber et al. 1996). However, the patients were also characterized for their NAb responses. This was measured as the geometric mean titer of the reciprocal dilution of late sera needed to neutralize $90 \%$ of the patient's own (autologous) virus.

\section{Simulation of V3 Evolution}

The population of HIV-1 virus particles (virions) within a patient is represented as a data vector in which each element contains the 96-nucleotide V3 sequence of one provirus (viral DNA integrated into host genome) (Fig. 1). A random number generator is used to mutate each nucleotide at an empirically estimated rate for HIV-1. Following mutation, nucleotide sequences are translated to amino acid sequences. Randomly chosen sequences (proviruses) are replicated with a probability proportional to their fitness, with fitness being determined by the form of selection. A constant population size is maintained by sampling (replicating) sequences with replacement until the desired next generation's population size is reached, thus completing one viral generation (Fig. 2).

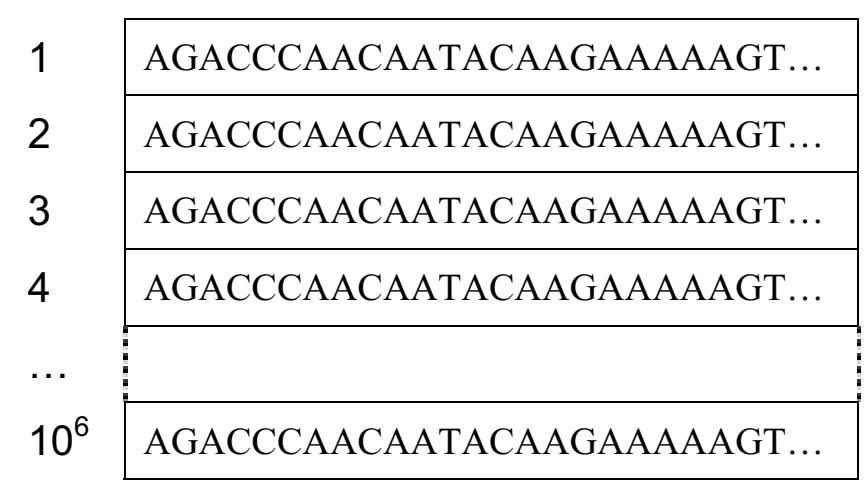

Figure 1. A V3 nucleotide sequence data vector representing a simulated viral population of $10^{6}$ virions within a patient.

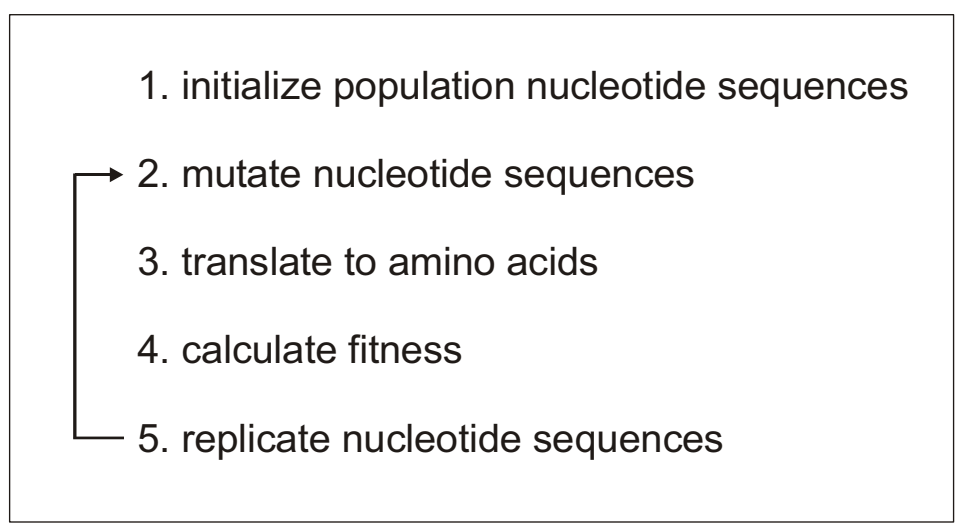

Figure 2. Flow of the simulation program. 


\section{Selection}

The form of selection imposed by NAbs is not well understood. Therefore, two plausible forms of selection were explored.

With negative frequency-dependent selection, the fitness of a viral variant is inversely proportional to its relative frequency. This type of selection may arise if the NAb response is directed mainly at the most common viral variants. Frequency-dependent selection was implemented by simply counting the numbers of each V3 variant and then assigning fitness based on each variant's relative frequency. This form of selection is described by $W=1-f^{b}$, where $W$ is the relative fitness (ranging from 0 to 1 ) of an amino-acid variant, $f$ is the variant's relative frequency, and $b$ shapes the fitness function (and is constrained to be $>0$ ) (Fig. 3A).

With negative age-dependent selection, the fitness of a viral variant declines exponentially with time (i.e. the number of viral generations) from the maximal value. This type of selection may arise if there is a time lag in the NAb response. The temporal dynamics of antibody responses are not well understood, but a time lag in the response has been shown for chimpanzees naturally infected with SIVcpz (Nyambi, Lewi et al. 1997) (Hahn, Shaw et al. 2000) and humans infected with HIV-1 (Albert, Abrahamsson et al. 1990; Bradney, Scheer et al. 1999). Age-dependent selection was implemented by maintaining a database containing each variant sequence and its age in the current population. This database was then referenced in the next generation to determine the ages of the then current variants. Fitnesses were assigned on the bases of variants' ages. Once a variant became extinct it was no longer maintained in the database, and if it reappeared in the population (as a result of mutation from another existing variant) it was assigned an age of zero just like any other new variant. Age-dependent selection was described with $W=\mathrm{e}^{-r t}$, where $t$ is a variant's age in generations and $r$ is the rate of exponential decline in fitness. As $r$ increases, fitness declines more precipitously with age (Fig. 3B). Estimates for $r$ are not available, but, based on preliminary studies, values from 0.001 to 0.01 were used.

Both forms of selection were expected to maintain genetic variation within a time point (diversity) because a variant's fitness declines as it becomes either common or long-lived.
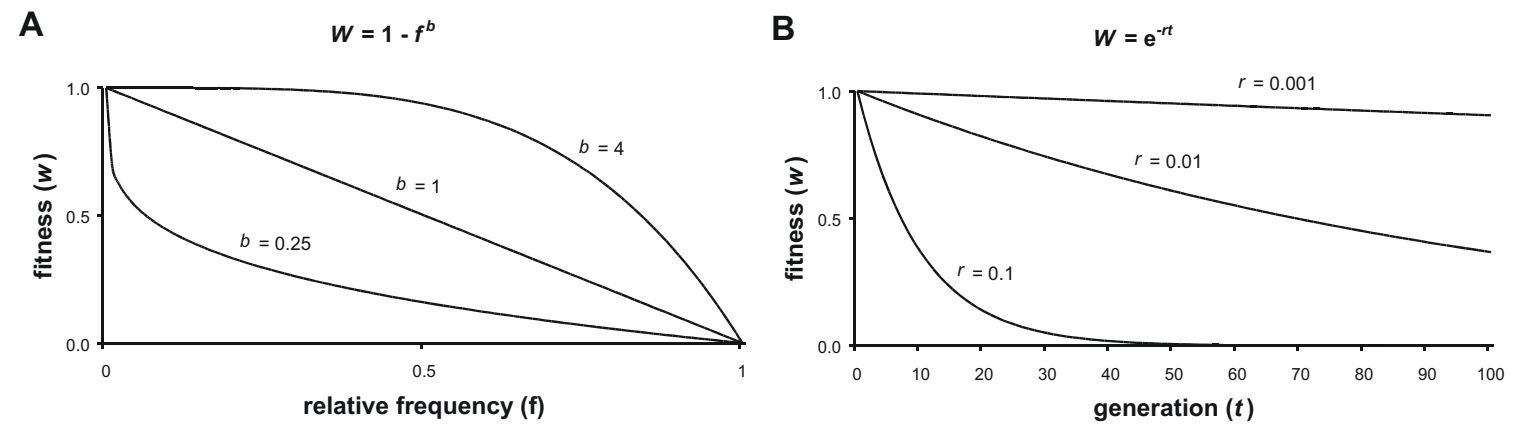

Figure 3. Forms of potential NAb selection. (A) Negative frequencydependent selection, in which the fitness of a variant is inversely proportional to its relative frequency. (B) Negative age-dependent selection, in which the fitness of a variant declines exponentially with its age (generations).

\section{Population and Replication Parameter Values}

Although the turnover rate of virions within a patient is on the order of $10^{8}$ (Wei, Ghosh et al. 1995) to $10^{10}$ (Perelson, Neumann et al. 1996) per day, the effective population size, that is, the number of virions that replicate, is much smaller: on the order of $10^{5}$ (Rouzine and Coffin 1999). A stable population of $10^{5}$ virions was used in simulations, and populations of $10^{4}$ and $10^{6}$ were used to test the sensitivity of the results to changes in population size. The overall mutation rate, 
including insertions and deletions of nucleotides, is estimated at $3.4 \times 10^{-5}$ per nucleotide per replication cycle, whereas the mutation rate for nucleotide substitutions exclusively is $2.41 \times 10^{-5}$ (Mansky and Temin 1995). A mutation rate of $10^{-5}$ was used in simulations, and mutation rates of $10^{-6}$ and $10^{-4}$ were used to test the model's sensitivity to the mutation rate. Mutation appears to be biased in HIV-1, with a preponderance of G-to-A mutations. Relative rates of mutation between nucleotides (Wiener 1996) were used to determine the specific mutations that occur. That is, given that a nucleotide site is to be mutated, the specific mutation will depend on the nucleotide present and the probability of that nucleotide mutating to each of the other three nucleotides. The length of the replication cycle (generation time) of HIV-1 has been estimated, using mathematical models, to be approximately 1.2 (Coffin 1995) to 2.6 (Perelson, Neumann et al. 1996) days per cycle. A more recent investigation, using coalescent theory, has estimated this value to be 1.2 (Rodrigo, Shpaer et al. 1999). Therefore, 1.2 days per cycle will be used in simulations.

\section{Simulation Runs}

Each run of a simulation consisted of 20 replicates of 1500 generations (60 simulated months) each. Because of the stochastic nature of Monte Carlo simulation, replication is necessary to estimate the stochastic error of estimates of means. A sample size of 20 replicates is generally considered the minimum required.

For each simulation replicate 20 nucleotide sequences were chosen randomly from the population every 150 generations (equivalent to six months) and written to an external file. The output from each replicate is meant to mimic the output from an intensive empirical study.

\section{Parallel Processing}

The simulation program, written in Fortran 90, was compiled using the IBM XL Fortran V. 6.1 compiler. Because of the naturally parallel structure of Monte Carlo simulations, simulation runs consisted of 20 replicate simulations processed in parallel. The single program multiple data programming model was implemented using the Message Passing Interface library of routines (Gropp, Lusk et al. 1994). Because of parallel processing, the pseudorandom number generator has to produce an independent stream of pseudorandom numbers for each process and each stream has to have a period (the number of pseudorandom numbers generated before the sequence of numbers is repeated) that is greater than the number of pseudorandom numbers required by the process. The Scalable Pseudorandom Number Generator (SPRNG) libraries (http://www.ncsa.uiuc.edu/Apps/CMP/RNG/RNG-home.html) were used to apply a Modified Lagged Fibonacci Generator with a period of approximately $2^{1310}$ and $2^{39617}$ distinct streams, which were sufficient for the number of generations and replicates simulated. A random number stream was generated for each simulation replicate, rather than each processor, to allow the exact same simulation to be reproduced (same seed value) regardless of the number of processors used. This was useful in debugging the program.

Simulations were run on an IBM RS/6000 SP. One simulation run consisting of 20 replicate simulations required approximately 360 total CPU hours for a population size of $10^{6}$ virions, 36 hours for $10^{5}$ virions, and 3.6 hours for $10^{4}$ virions. A maximum of $180 \mathrm{MB}$ of runtime memory were required to store information in various population vectors used during the most memory-intensive simulations (age-dependent selection) of the largest population size $\left(10^{6}\right.$ virions).

\section{Results and Discussion}

\section{Empirical Patterns}

$d_{\mathrm{N}}$ reflects amino acid diversity and can also be used to detect selection for amino acid replacement (positive selection). In these terms, the prediction is that $d_{\mathrm{N}}$ will be higher in patients 
with a strong antibody response than in patients with a weak response and that only in strong responders will $d_{\mathrm{N}}$ exceed $d_{\mathrm{S}}$. The divergence of $\mathrm{V} 3$ in each patient was measured by estimating $d_{\mathrm{S}}$ and $d_{\mathrm{N}}$, as described above, from all possible pairwise comparisons between sequences from one time point sample of sequences and the initial sample. $d_{\mathrm{N}}$ exceeded $d_{\mathrm{S}}$ significantly only for patient P4, the patient with the strongest NAb response (Fig. 4). The diversity of V3 in each patient was measured by estimating $d_{\mathrm{S}}$ and $d_{\mathrm{N}}$ from pairwise comparisons among sequences within each time point. Again, $d_{\mathrm{N}}$ exceeded $d_{\mathrm{S}}$ significantly only for patient P4 (Fig. 5).
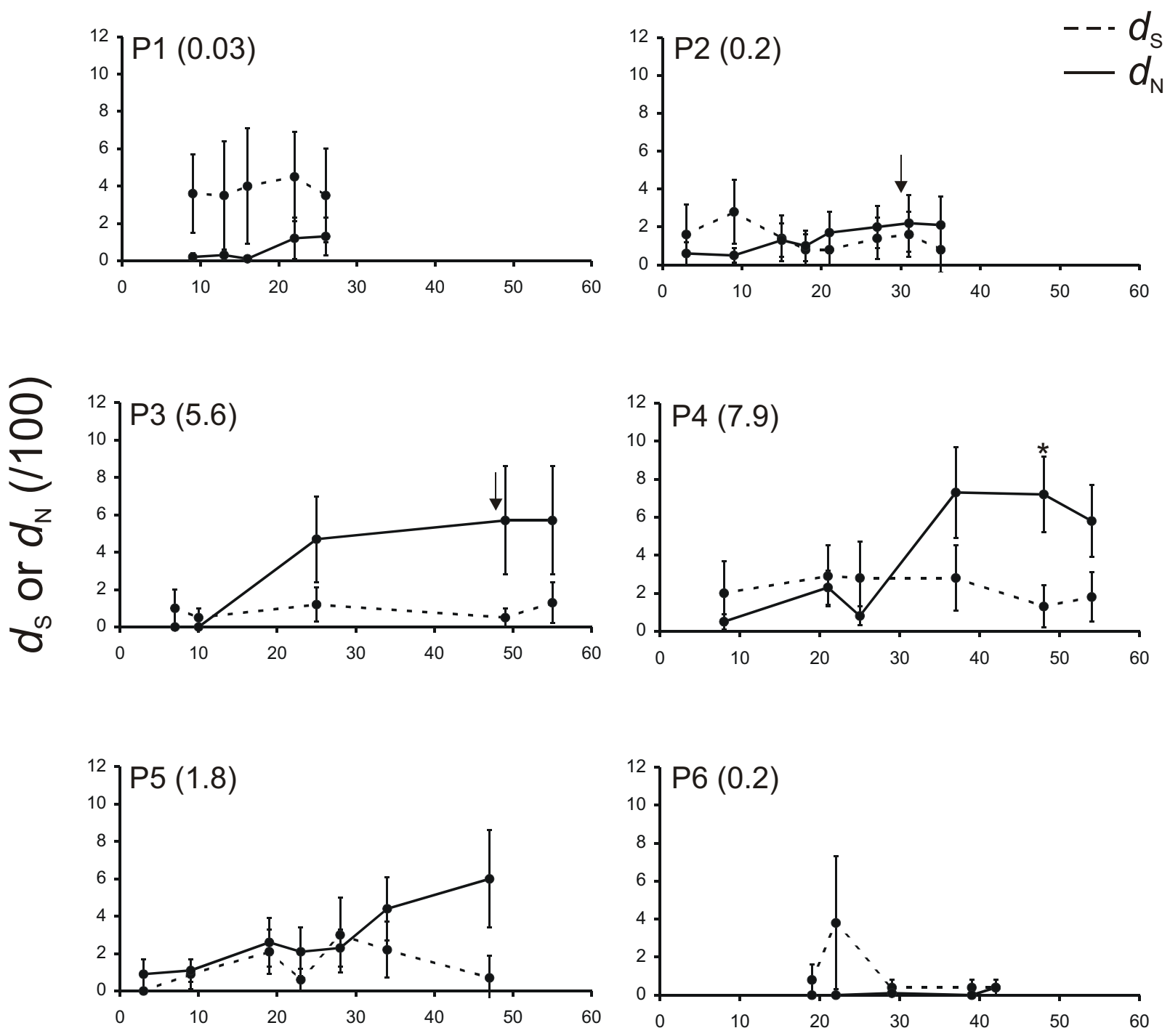
Number of months post infection

Figure 4. Divergence $d_{\mathrm{S}}$ and $d_{\mathrm{N}}( \pm 1 \mathrm{SE})$ plotted against the number of months post infection. NAb responses (see text) are given in parentheses after the patient's identification. Arrows mark the start of antiviral therapy (zidovudine). Numbers of sequences sampled in each time-point in chronological order: P1 (11, 8, 8, 8, 7); P2 $(5,6,7,4,5,7,5,2)$; P3 $(8,6,6,5,5)$; P4 (11, 7, 7, 7, 8, 6); P5 $(8,9,10,7,11,11,6)$; P6 $(10,6$, $10,3,10)$. Tests of $d_{\mathrm{S}}=d_{\mathrm{N}}: * P<0.05$. 

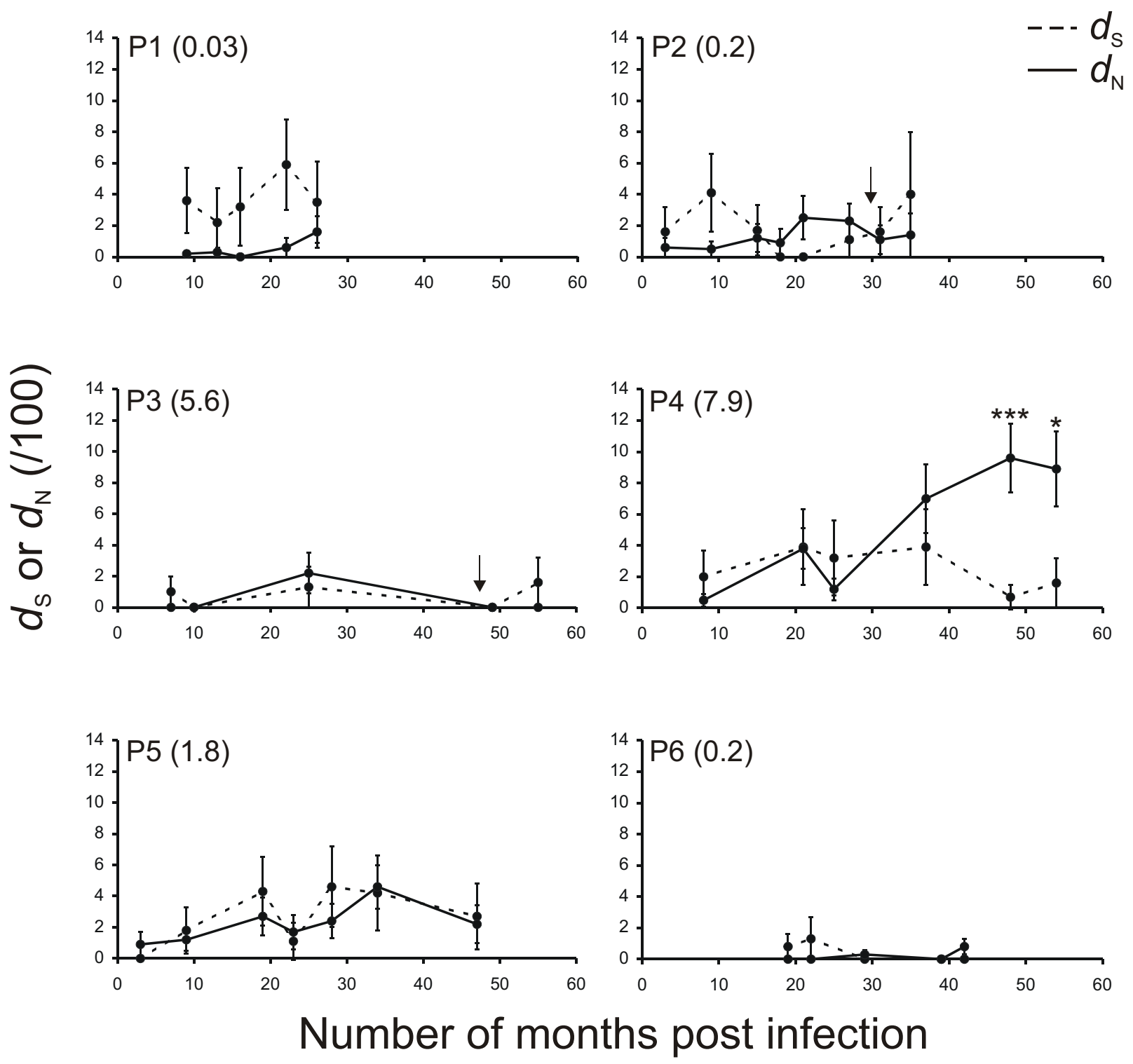

Figure 5. Diversity $d_{\mathrm{S}}$ and $d_{\mathrm{N}}( \pm 1 \mathrm{SE})$ plotted against the number of months post infection. Symbols and sample sizes are as in Figure 2. Tests of $d_{\mathrm{S}}=d_{\mathrm{N}}: * P<0.05 ; * * * P<0.001$.

\section{Model Test}

We tested the routines fundamental to the model, that is, those implementing mutation and replication (and hence pseudorandom number generation), in the absence of selection by comparing simulated gene diversities to expected gene diversities. Average gene diversity $(h)$ is calculated as

$$
\text { h }{ }_{i=1}^{q} x_{\mathrm{i}}^{2},
$$

where $x_{i}$ is the frequency of the $i^{\text {th }}$ of $q$ alleles (amino-acid variants). The average gene diversity expected under strict neutrality (no selection) and at equilibrium for an infinite-alleles model (infinite number of amino-acid sites) $(E(\hat{H}))$ is 


$$
E(\hat{H}) \frac{2 N v}{12 N v}
$$

(Nei 1987, p. 377), where $N$ is the haploid effective population size (stable number of virions) and $v$ is the per-sequence mutation rate. The per-sequence mutation rate is calculated as $v=\mu \mathrm{x}$ $n_{\mathrm{N}}$, where $\mu$ is the per nucleotide mutation rate and $n_{\mathrm{N}}$ is the number of nonsynonymous (amino acid changing) nucleotide sites in the simulated sequence. Simulations were run without selection for 5,000 generations or until equilibrium was reached ( $h$ remained constant). The variance of $h$ was estimated for each time point from 20 replicate simulations. The equilibrium value of $h$ was compared to the expected value. Tests were conducted with parameter values $N=10^{4}, 10^{5}$, and $\mu$ $=10^{-4}, 10^{-5}$. In all cases, observed average gene diversities at equilibrium matched expected gene diversities (Fig. 6).

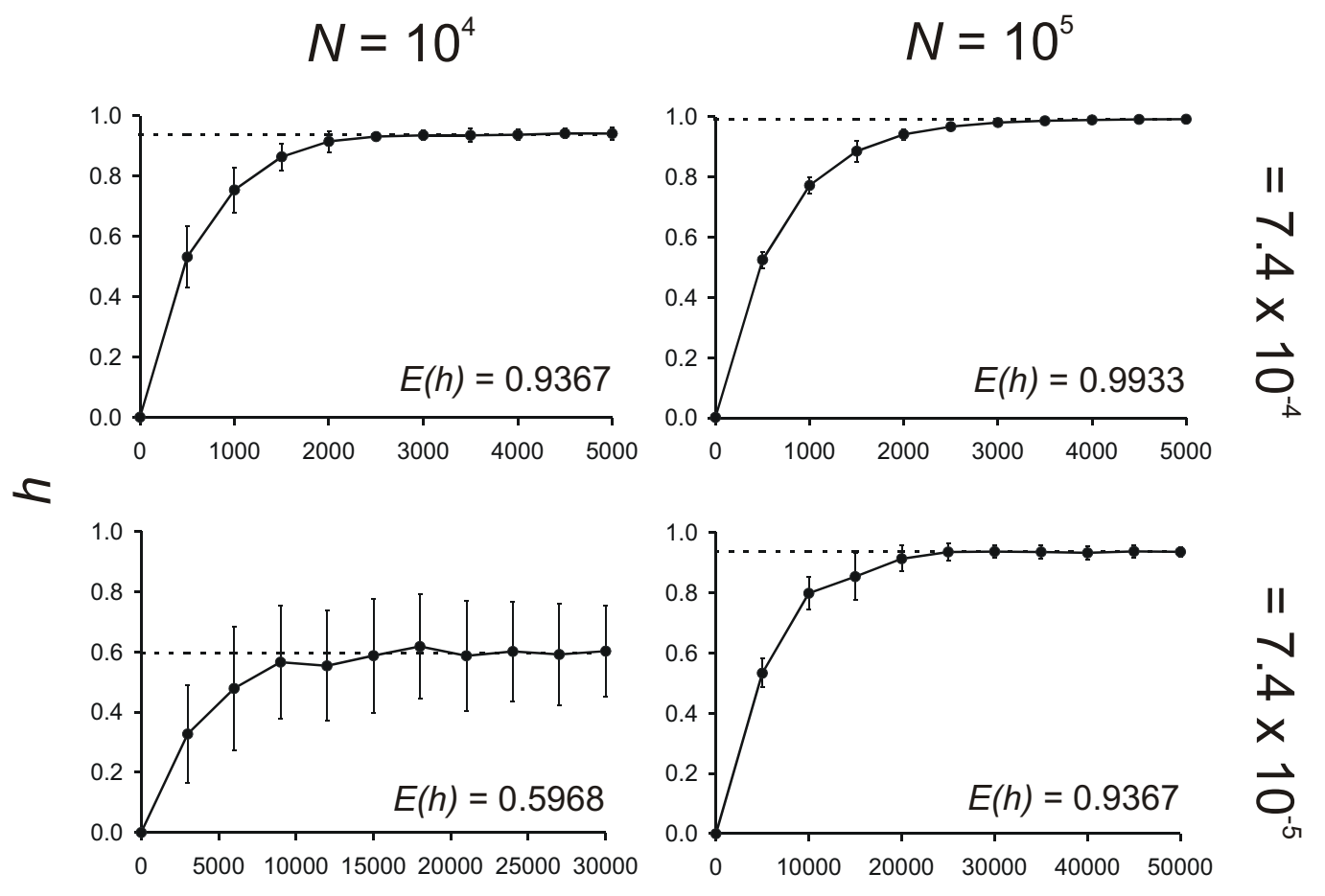

\section{Generation}

Figure 6. Average gene diversity $(h)( \pm 1 \mathrm{SE})$ plotted against viral generation for two effective population sizes $(N)$ and two per-sequence mutation rates $(v)$ from 20 replicate simulations. Dotted reference lines show the expected gene diversity $(E(h))$ under strict neutrality at equilibrium for the infinite-alleles model.

\section{Simulated Patterns}

With frequency-dependent selection, diversity $d_{\mathrm{N}}$ rose quickly and then remained relatively constant and higher than $d_{\mathrm{S}}$ (Fig. 7A). With age-dependent selection, there was a lag in the increase in $d_{\mathrm{N}}$, but then a long period of increase (Fig. 7B). The latter result more closely mimics the empirical result from the patient with a strong NAb response (P4) (Fig. 5). These results suggest that strong NAb selection is variant age-dependent, and imply a time lag in the NAb response. 
A

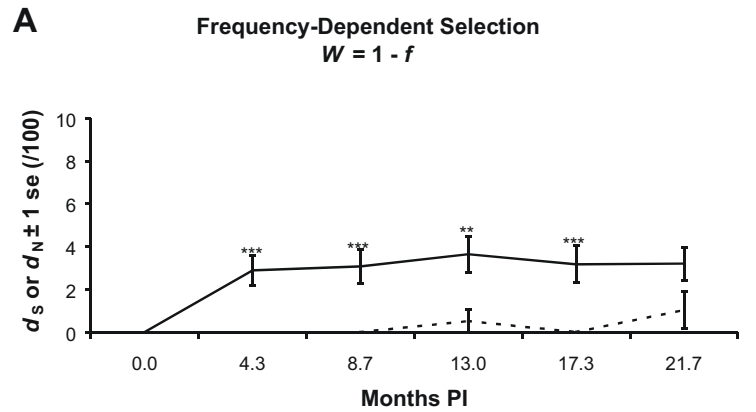

B

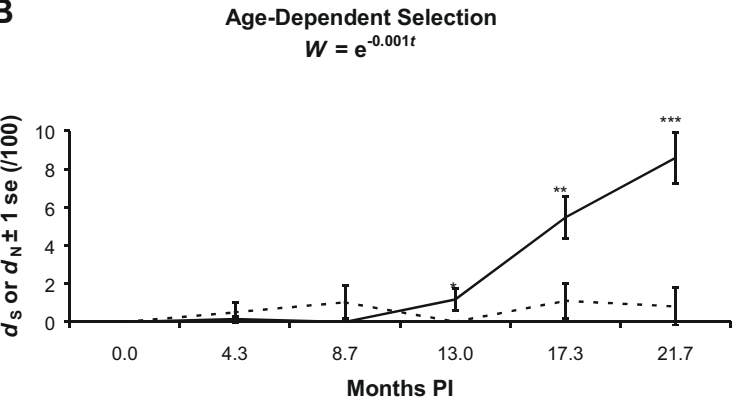

Figure 7. Results from one replicate simulation of NAb selection. Diversity $d_{\mathrm{S}}$ (dashed line) and $d_{\mathrm{N}}$ (solid line) for 20 simulated V3 sequences sampled at various times post-infection (PI). Z-tests: ${ }^{*} P<$ $0.05 ; * * P<0.01 ; * * * P<0.001$. (A) Negative frequency-dependent selection with a linear fitness function. (B) Negative age-dependent selection with $r=0.001$.

\section{Conclusion}

These results support the hypothesis that HIV-1 evolves in response to selection by NAbs. The empirical data show evidence of positive selection of $\mathrm{V} 3$ only in the patient with the strongest $\mathrm{NAb}$ response. The pattern of change in diversity $d_{\mathrm{N}}$ in this patient is better simulated with negative age-dependent selection than with negative frequency-dependent selection. This implies that there is a time lag in the production of antibodies to newly emerged V3 variants. This conclusion is consistent with results from studies of the tempo of antibody selection of SIVcpz infecting chimpanzees (Nyambi, Lewi et al. 1997) and HIV-1 infecting humans (Albert, Abrahamsson et al. 1990; Bradney, Scheer et al. 1999).

\section{Literature Cited}

Albert, J., B. Abrahamsson, et al. (1990). "Rapid development of isolate-specific neutralizing antibodies after primary HIV-1 infection and consequent emergence of virus variants which resist neutralization by autologous sera." Aids 4(2): 107-12.

Bonhoeffer, S., E. C. Holmes, et al. (1995). "Causes of HIV diversity." Nature 376(6536): 125.

Bradney, A. P., S. Scheer, et al. (1999). "Neutralization escape in human immunodeficiency virus type 1-infected long-term nonprogressors." J Infect Dis 179(5): 1264-7.

Cheng-Mayer, C., A. Brown, et al. (1999). "Selection for neutralization resistance of the simian/human immunodeficiency virus SHIVSF33A variant in vivo by virtue of sequence changes in the extracellular envelope glycoprotein that modify N-linked glycosylation." $\underline{\mathrm{J}}$ Virol 73(7): 5294-300.

Coffin, J. M. (1995). "HIV population dynamics in vivo: implications for genetic variation, pathogenesis, and therapy." Science 267(5197): 483-9.

da Silva, J. and A. L. Hughes (1999). "Molecular phylogenetic evidence of cytotoxic T lymphocyte (CTL) selection on human immunodeficiency virus type 1 (HIV-1)." $\underline{\text { Mol }}$ Biol Evol 16(10): 1420-1422.

Desrosiers, R. C. (1999). "Strategies used by human immunodeficiency virus that allow persistent viral replication." Nat Med 5(7): 723-5.

Etemad-Moghadam, B., Y. Sun, et al. (1999). "Determinants of neutralization resistance in the envelope glycoproteins of a simian-human immunodeficiency virus passaged in vivo." $\underline{\mathrm{J}}$ Virol 73(10): 8873-9. 
Evans, D. T., D. H. O'Connor, et al. (1999). "Virus-specific cytotoxic T-lymphocyte responses select for amino-acid variation in simian immunodeficiency virus Env and Nef." Nature Medicine 5(11): 1270-1276.

Fouts, T. R., J. M. Binley, et al. (1997). "Neutralization of the human immunodeficiency virus type 1 primary isolate JR-FL by human monoclonal antibodies correlates with antibody binding to the oligomeric form of the envelope glycoprotein complex." J Virol 71(4): 2779-85.

Gropp, W., E. Lusk, et al. (1994). Using MPI: Portable Parallel Programming with the MessagePassing Interface. Cambridge, Mass., The MIT Press.

Haefner, J. W. (1996). Modeling Biological Systems: Principles and Applications. New York, NY, Chapman \& Hall.

Hahn, B. H., G. M. Shaw, et al. (2000). "AIDS as a Zoonosis: Scientific and Public Health Implications." Science 287: 607-614.

Halapi, E., T. Leitner, et al. (1997). "Correlation between HIV sequence evolution, specific immune response and clinical outcome in vertically infected infants." Aids 11(14): 170917.

Inouye, P., E. Cherry, et al. (1998). "Neutralizing antibodies directed against the V3 loop select for different escape variants in a virus with mutated reverse transcriptase (M184V) than in wild-type human immunodeficiency virus type 1." AIDS Res Hum Retroviruses 14(9): $735-40$.

Jukes, T. H. and C. R. Cantor (1969). Evolution of protein molecules. Mammalian Protein Metabolism. H. N. Munro. New York, Academic Press: 21-132.

Kumar, S., K. Tamura, et al. (1993). MEGA: Molecular Evolutionary Genetic Analysis, version 1.01. University Park, The Pennsylvania State University.

Kwong, P. D., R. Wyatt, et al. (1998). "Structure of an HIV gp120 envelope glycoprotein in complex with the CD4 receptor and a neutralizing human antibody." Nature 393(6686): 648-59.

Liu, S. L., T. Schacker, et al. (1997). "Divergent patterns of progression to AIDS after infection from the same source: human immunodeficiency virus type 1 evolution and antiviral responses." J Virol 71(6): 4284-95.

Mansky, L. M. and H. M. Temin (1995). "Lower in vivo mutation rate of human immunodeficiency virus type 1 than that predicted from the fidelity of purified reverse transcriptase." J Virol 69(8): 5087-94.

McKnight, A., R. A. Weiss, et al. (1995). "Change in tropism upon immune escape by human immunodeficiency virus." J Virol 69(5): 3167-70.

McMichael, A. J. and R. E. Phillips (1997). "Escape of human immunodeficiency virus from immune control." Annu Rev Immunol 15: 271-96.

Nei, M. (1987). Molecular evolutionary genetics. New York, Columbia Univesity Press.

Nei, M. and T. Gojobori (1986). "Simple methods for estimating the numbers of synonymous and nonsynonymous nucleotide substitutions." Mol Biol Evol 3(5): 418-26.

Nei, M. and L. Jin (1989). "Variances of the average numbers of nucleotide substitutions within and between populations." Mol Biol Evol 6: 418-426.

Nyambi, P. N., P. Lewi, et al. (1997). "Study of the dynamics of neutralization escape mutants in a chimpanzee naturally infected with the simian immunodeficiency virus SIVcpz-ant." $\underline{J}$ Virol 71(3): 2320-30.

Nyambi, P. N., H. A. Mbah, et al. (2000). "Conserved and exposed epitopes on intact, native, primary human immunodeficiency virus type 1 virions of group M." J Virol 74(15): 7096-107.

Perelson, A. S., A. U. Neumann, et al. (1996). "HIV-1 dynamics in vivo: virion clearance rate, infected cell life-span, and viral generation time." Science 271(5255): 1582-6. 
Profy, A. T., P. A. Salinas, et al. (1990). "Epitopes recognized by the neutralizing antibodies of an HIV-1-infected individual." J Immunol 144(12): 4641-7.

Rodrigo, A. G., E. G. Shpaer, et al. (1999). "Coalescent estimates of HIV-1 generation time in vivo." Proc Natl Acad Sci U S A 96(5): 2187-91.

Rouzine, I. M. and J. M. Coffin (1999). "Linkage disequilibrium test implies a large effective population number for HIV in vivo." Proc Natl Acad Sci U S A 96(19): 10758-63.

Schonning, K., B. Jansson, et al. (1996). "Rapid selection for an N-linked oligosaccharide by monoclonal antibodies directed against the V3 loop of human immunodeficiency virus type 1." J Gen Virol 77(Pt 4): 753-8.

Speck, R. F., K. Wehrly, et al. (1997). "Selective employment of chemokine receptors as human immunodeficiency virus type 1 coreceptors determined by individual amino acids within the envelope V3 loop." J Virol 71(9): 7136-9.

Spenlehauer, C., S. Saragosti, et al. (1998). "Study of the V3 loop as a target epitope for antibodies involved in the neutralization of primary isolates versus T-cell-line-adapted strains of human immunodeficiency virus type 1." J Virol 72(12): 9855-64.

Viscidi, R. P. (1999). HIV evolution and disease progression via longitudinal studies. The Evolution of HIV. K. A. Crandall. Baltimore, John Hopkins University Press.

Wei, X., S. K. Ghosh, et al. (1995). "Viral dynamics in human immunodeficiency virus type 1 infection." Nature 373(6510): 117-22.

Weiss, R. A. (1996). "HIV receptors and the pathogenesis of AIDS." Science 272(5270): 1885-6.

Wiener, P. (1996). "Inferring frequency dependent selection from the molecular evolution of a rapidly evolving virus: a theoretical investigation." Proc R Soc Lond B Biol Sci 263(1375): 1283-9.

Wolinsky, S. M., B. T. Korber, et al. (1996). "Adaptive evolution of human immunodeficiency virus-type 1 during the natural course of infection." Science 272(5261): 537-42.

Wolinsky, S. M. and G. H. Learn (1999). Levels of diversity within and among host individuals. The Evolution of HIV. K. A. Crandall. Baltimore, John Hopkins University Press.

Yoshida, K., M. Nakamura, et al. (1997). "Mutations of the HIV type 1 V3 loop under selection pressure with neutralizing monoclonal antibody NM-01." AIDS Res Hum Retroviruses 13(15): 1283-90. 